\title{
Pedicled Latissimus Dorsi Kiss Flap for the Reconstruction of Large Chest Wall Defect After Mastectomy
}

\author{
Shengchao Huang ${ }^{1} \cdot$ Pu Qiu$^{1}$ - Jianwen $\mathrm{Li}^{1}$ • Weizhang Chen ${ }^{1} \cdot$ Zhongzeng Liang $^{1} \cdot$ Kangwei Luo $^{1}$ - Baoyi Huang ${ }^{1}$. \\ Liyan $\mathrm{Yu}^{1} \cdot$ Zeming Yan $^{1} \cdot$ Yuanqi Zhang ${ }^{1}$
}

Received: 18 November 2020 / Accepted: 4 September 2021 / Published online: 25 November 2021

(c) The Author(s) 2021

\begin{abstract}
To discover the utility of pedicled latissimus dorsi kiss flap for the reconstruction of chest wall defect after mastectomy. This study was a systemic analysis of 12 female patients with breast tumors who were treated at Affiliated Hospital of Guangdong Medical University from January 2018 to December 2019. Among them, three patients had malignant lobular breast tumors, and nine patients had locally advanced breast cancer. After extensive resection of the primary tumor, the chest wall skin, and soft tissue, a large defect was left in the chest wall of each patient. Based on the design and structure of the kiss flap, two semicircular flaps of equal diameter were designed in the latissimus dorsi region, and their blood supply was retained from the same vascular trunk. Two flaps were transferred to the chest wall through a subcutaneous tunnel, and the incision in the donor area was sutured directly. Finally, two equal semicircle flaps were adjusted to fit the defect and then fixed on the chest wall. Referred to the design of the kiss flap, the area of the latissimus dorsi was increased to cover a larger chest wall defect. We have used this flap to reconstruct chest wall defects on twelve patients. Their age ranged from 24 to 62 . The largest defect was $20 \times 12 \mathrm{~cm}$, and the smallest defect was $15 \times 10 \mathrm{~cm}$ in diameter. Postoperative follow-up time was 5-9 months (mean time: 6.2 months): Follow-up observations demonstrated that all the flaps were healed well without edema or extravasation and donor area of all cases was closed well. In addition, no local recurrence or distant metastasis was observed in all patients.
\end{abstract}

Keywords Breast tumors $\cdot$ Locally advanced breast cancer $\cdot$ Lobular tumor $\cdot$ Latissimus dorsi kiss flap $\cdot$ Chest wall reconstruction

\section{Introduction}

With the promotion and popularization of breast cancer screening, most of the breast cancer patients could be diagnosed early and received standardized treatment. And these patients had a good prognosis. Unfortunately, some patients did not seek medical care until they developed a larger breast tumor, because they did not pay attention to it. The majority of them was diagnosed with locally advanced breast cancer or malignant breast phyllodes tumors. In underdeveloped areas of China, these types of patient were more common.

Shengchao Huang and Pu Qiu are co-first authors.

Yuanqi Zhang

3148319@qq.com

1 Department of Breast Surgery, Affiliated Hospital of Guangdong Medical University, 53 Renmin Avenue South, Zhanjiang 524001, China
To reduce the local recurrence of the tumor, extensive resection was typically necessary for such patients [1]. Reconstruction of the defect on the chest wall after mastectomy had been problematic for breast surgeons. The latissimus dorsi kiss flap was based on this concept to improve the traditional latissimus dorsi musculocutaneous flap [2-4] and encompassed two semicircle flaps that were supplied by the same blood vessel. After cutting, two semicircle flaps were pulled along the subcutaneous tunnel to the recipient area and spliced to repair the defect. After the kiss flap had been transferred, the surrounding skin and soft tissue of the donor area were closed through geometric stitching without skin grafts. Because the kiss flap did not require vascular anastomosis, this flap cloud be used by a breast surgeon to repair large chest wall defects in breast centers where microvascular anastomosis was not available. This report reviewed and analyzed 12 patients who underwent this procedure at Affiliated Hospital of Guangdong Medical University from January 2018 to December 2019. Three patients had malignant 
lobular breast tumors, and nine patients had locally advanced breast cancer. Their information of preoperative design, tumor resection, repair methods of the chest wall defect, and postoperative recovery was collected to summarize lessons of our department about applying pedicled latissimus dorsi kiss flap to treat chest wall defect after breast tumor resection.

\section{Patients and Methods}

\section{Patient Characteristics}

There were twelve breast cancer patients who had been treated at Affiliated Hospital of Guangdong Medical University from January 2018 to December 2019. Their age ranged from 24 to 62 , with an average age of 52.5 years. The diameter of the tumor ranged from 8 to $18 \mathrm{~cm}$. All patients had underwent hollow needle puncture prior to surgery: three patients were pathologically diagnosed with malignant breast lobular tumors, and nine patients were pathologically diagnosed with locally advanced breast cancer. No distant cancer metastasis was observed as determined by preoperative examination of the breast, bilateral axillary color Doppler ultrasound, collarbone region color Doppler ultrasound, mammary molybdenum target, tumor indicators, multi-site CT, and whole-body bone scans. Three cases had outer upper quadrant of left breast; one case had inner upper quadrant of left breast; two cases had outer lower quadrant of left breast; one case had inner lower quadrant of left breast; one case had outer upper quadrant of right breast; one case had inner upper quadrant of the right breast; and two cases had outer lower quadrant of the right breast. Among these patients, chest wall invasion was observed in three patients; skin invasion observed in seven patients and the tumor mass was greater than $5 \mathrm{~cm}$, but no tumor invasion of the skin or chest was observed in two patients. The largest defect on chest wall was $20 \times 12 \mathrm{~cm}$, and the smallest defect was $15 \times 10 \mathrm{~cm}$ in diameter, with an average defect area of $16.9 \mathrm{c} \times 11.4 \mathrm{~cm}$. After surgery, patients were administered standardized systematic treatment (Table 1).

\section{Surgical Methods}

\section{Preoperative Design}

In the supine position, the tumor size was measured and its boundaries was marked. And in the lateral position, with the assistance of bedside ultrasound Doppler, the direction of the main trunk of the thoracic dorsal artery was located and marked (when possible, CTA was used to reconfirm the vascular activity of the thoracic dorsal artery). The distance between the supply vessels of the latissimus dorsi musculocutaneous flap and the tumor center point of chest wall was measured, and the location of cutaneous island of latissimus dorsi flap was confirmed. In the design of the kiss flap, the position of the vessel pedicle of the latissimus dorsi was considered, and then, the latissimus dorsi flap was divided into two semicircular flaps (flap A and flap B) and labeled. The diameters of two semicircular flaps should intersect at one point, and their cross angle should be between $60^{\circ}$ and $90^{\circ}$, which is sufficient for direct sutures at the donor site without tension.

\section{Resection of the Breast Tumor}

During surgery, the patient was placed in the supine position with the upper limb abduction at an abduction of $90^{\circ}$. The primary tumor was first excised using an enlarged resection, which was at least $2 \mathrm{~cm}$ larger than the tumor boundary. When separating the lateral margin of the mammary gland and dissecting the axillary lymph nodes, it was important to protect the dorsal thoracic vessels and nerves during the procedure. If the skin or chest muscles had been involved, radical resection was performed. The edges of the excised tumor were then pathologically diagnosed to determine the presence or absence of residual tumor cells. After resection of the tumor, the wound was measured intraoperatively and then covered with a sterile membrane. Then, the patient was adjusted on the lateral decubitus position. If the size of the wound is similar to that before operation, there is no need to adjust the size of the flap. However, if the size difference is significant, the flap design may need to be adjusted.

\section{Separation of the Kiss Flaps}

The skin of the back was cut along the pre-designed marked lines to generate the two semicircular flaps. The two labeled semicircular flaps were separated using an electric knife. Attention was paid to protect the blood supply of the flap. In addition, the fat on the surface of the muscle was retained as far as possible. But both flaps were kept attached to the latissimus dorsi. After that, the isolation of the latissimus dorsi muscle boundary was performed. The latissimus dorsi was then positioned in the direction of the thoracic dorsal vessels. An ultrasonic scalpel was used to separate the space between the serratus anterior and latissimus dorsi, and then, the thoracic dorsal vessels were located and dissected. Along the thoracic dorsal vessels, an ultrasound knife was used to dissociate the thoracic dorsal vessels from the muscle bundle, which was to increase the length of the vascular pedicle and increase its range of movement. At this time, two semicircular flaps were formed on the latissimus dorsi muscle, known as the kiss flap. It was transferred to the chest defect along the subcutaneous tunnel, noting for any distortion of the vessel pedicle. After flushing and hemostasis of 


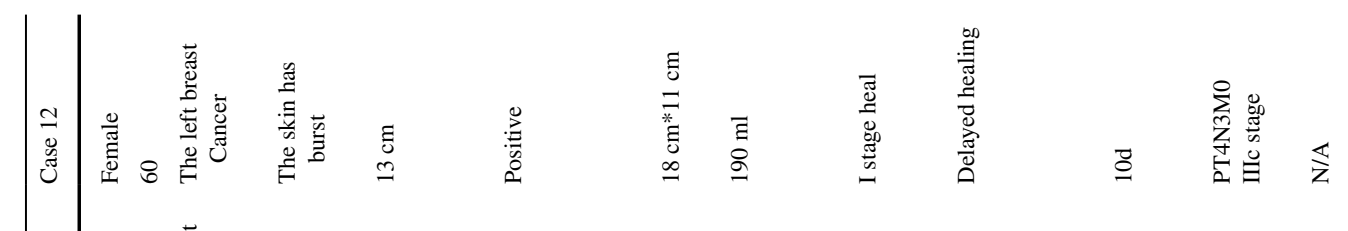

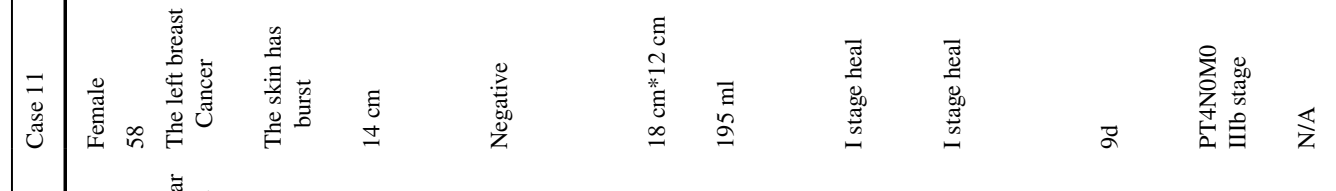

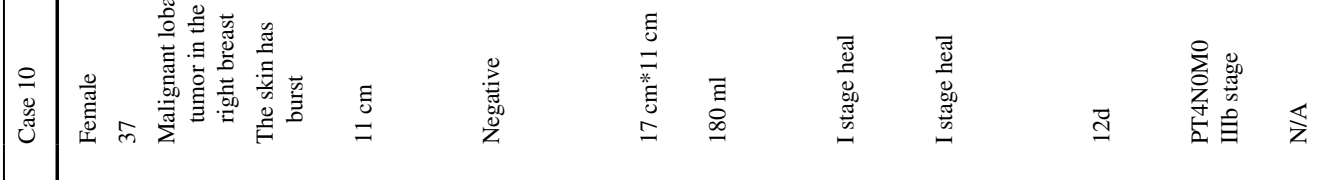

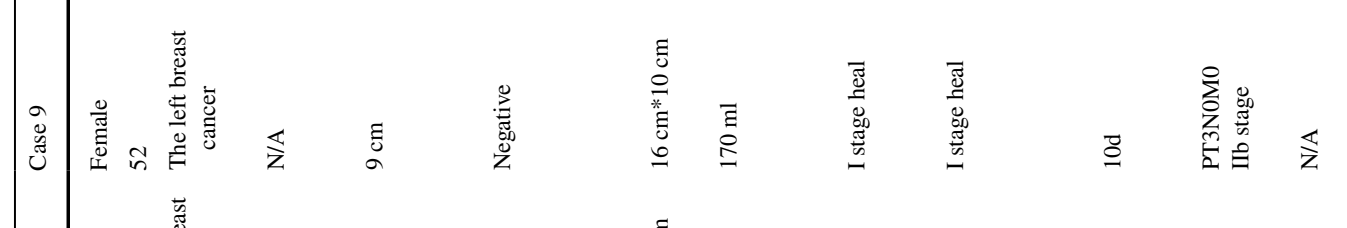

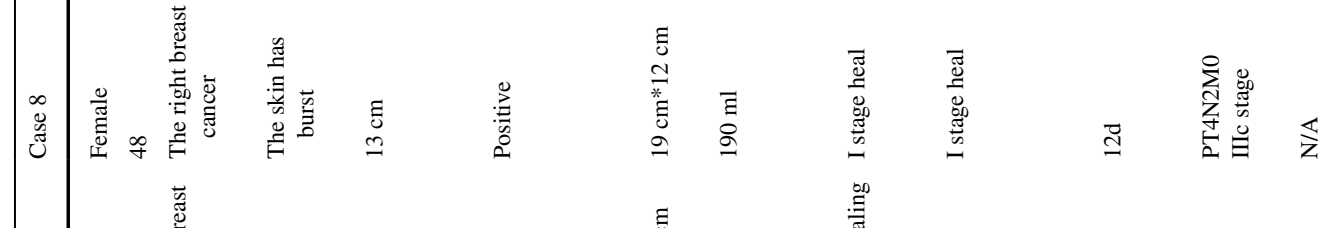

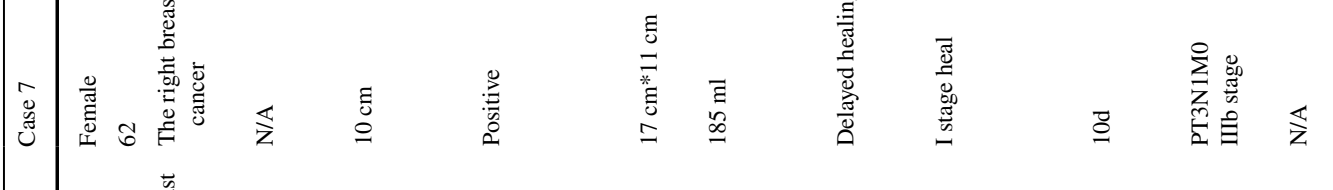

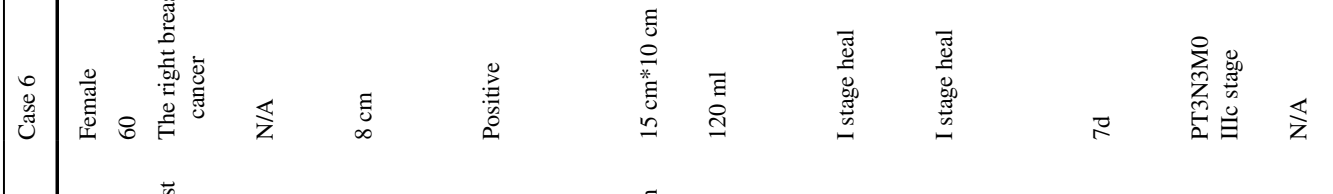

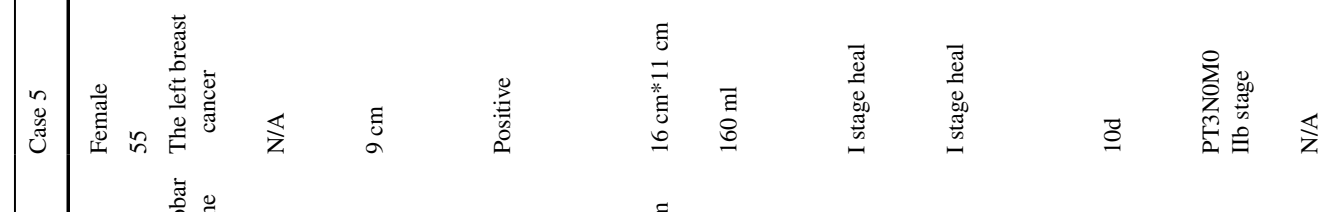

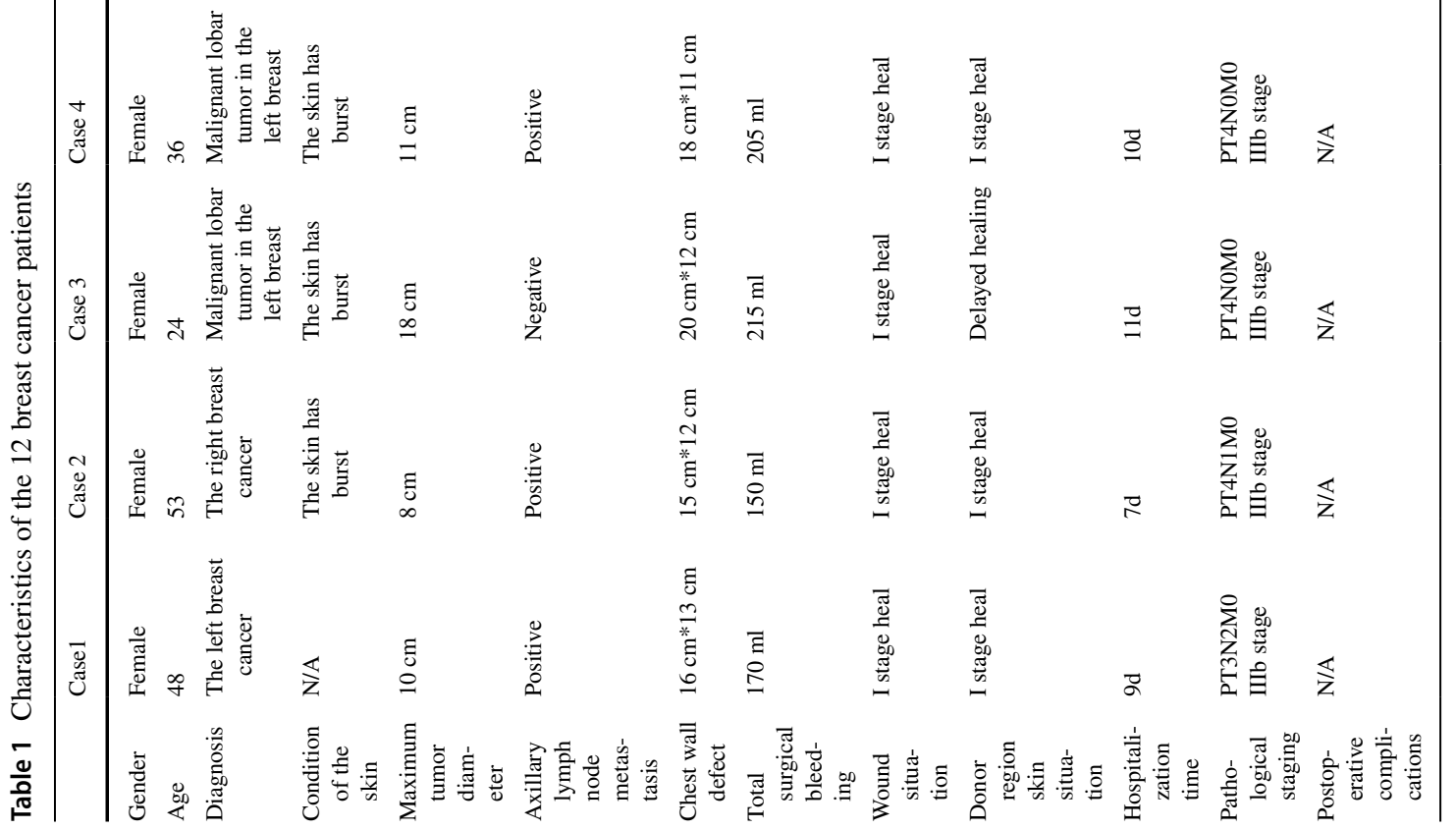


the back supply area, a drainage tube was placed, and the incision was closed.

\section{Flap Fixation}

Finally, the patient was adjusted in the supine position with the upper limbs maintained at an abduction of $90^{\circ}$. The chest wall was then rinsed again with normal saline. Based on the size and position of the wound, the splicing position of the two small semicircular flaps was adjusted in order to ensure that the myocutaneous flap was free of tension in the chest area. Then, a second examination confirmed no torsion of the pedicle. Afterward, a drainage tube was placed in the chest and the axilla. The subcutaneous tissue was subtracted sutured, and the intradermal tissue was sutured intermittently (Fig. 1a, b).

\section{Typical Patient Case}

A 45-year-old lady was hospitalized after the discovery of a left breast mass for about a year.

Physical Examinations Indicated A palpable mass about $5.0 \times 3.0 \mathrm{~cm}$ in the left breast from 7 to $11 \mathrm{o}$ 'clock. The skin of the left breast was red and swollen in several places. Multiple enlarged lymph nodes were observed in the left axilla with a hard texture and unclear boundary.

Biopsy Pathological Report A non-specific invasive ductal carcinoma on the left breast.

\section{PET-CT Examination No distant metastasis.}

The diagnosis and TNM staging were CT4N2M0 IIIc stage (negative breast cancer type).

Neoadjuvant regimens with 4 cycles "Docetaxel+adriamycin + Cyclophosphamide" were given, but the efficacy was evaluated as progressive disease (PD).
After MDT discussions, we decided to apply the latissimus dorsi kiss flap to cover the chest wall defect after radical breast cancer treatment. Intraoperatively, we removed the primary breast tumor and enlarged lymph nodes. The size of the left chest wall defect was $17 \times 11 \mathrm{~cm}$. Latissimus dorsi kiss flap was used to repair the defect on chest wall. The total blood loss during surgery was $180 \mathrm{ml}$. The postoperative pathological report indicated a non-specific invasive carcinoma of the left breast and no metastasis in the left axilla lymph nodes. Postoperative recovery was uneventful with no flap necrosis (Fig. 2a, f).

Ten days after surgery, the patient was discharged from the hospital. One week after discharge, intensive three cycles "Vinorelbine + cisplatin" chemotherapy were given and then followed by radiation therapy. During postoperative radiotherapy and chemotherapy, there were no signs of necrosis, edema, and congestion of kiss flap. Intensive capecitabine therapy was then given, and the patient was followed up 9 months later: the appearance of the flap was good, and no local recurrence or distant metastasis was observed.

\section{Results}

There were twelve patients who were treated with the latissimus dorsi kiss flap after tumor resection. The average surgical blood loss of twelve cases was $177.5 \mathrm{ml}$. The average length of hospitalization was 9.75 days. The follow-up period ranged from 5 to 9 months (mean time: 6.2 months). Follow-up results demonstrated that all the flaps grew well without edema or extravasation, and no local recurrence or distant metastasis was observed in all patients. Moreover, all donor region incisions healed well. All patients were administered standardized combination therapy.
Fig. 1 a Preoperative flap design b Flap suture
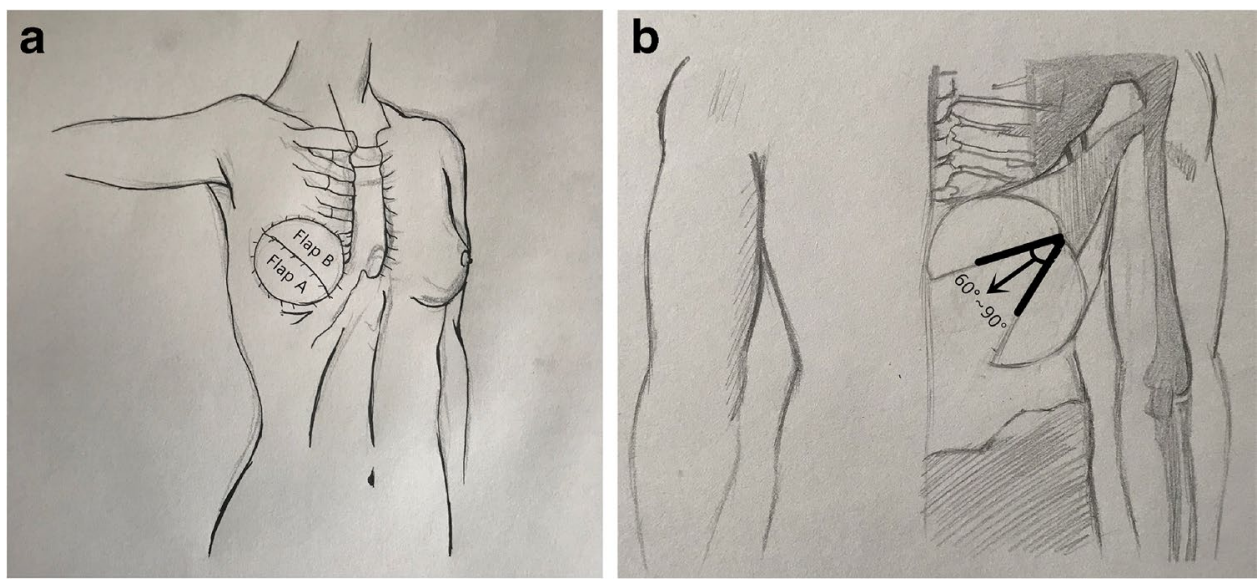
Fig. 2 a-f Preoperative and intraoperative situations in case
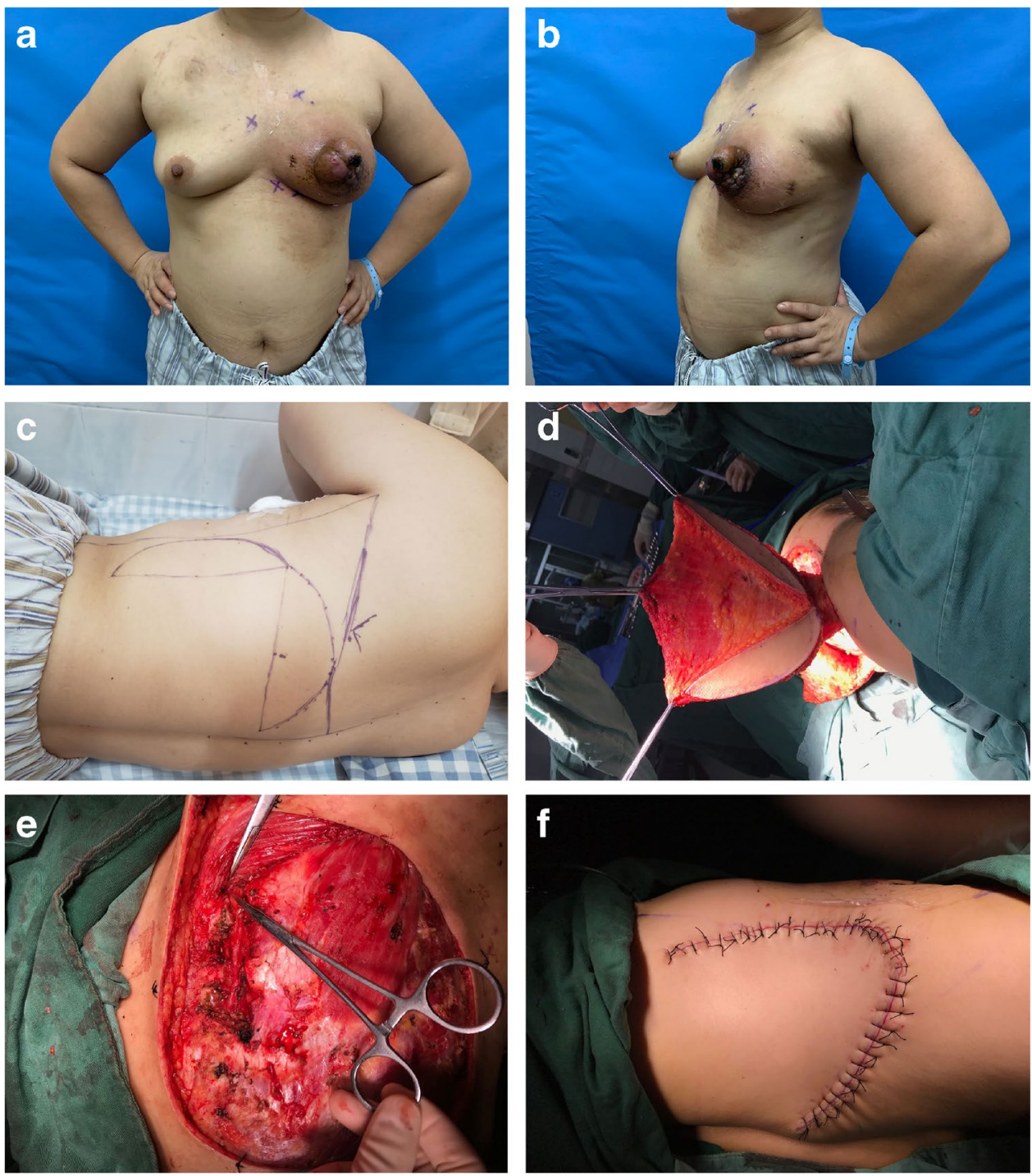

\section{Discussion}

As the tumor invades the skin, these patients developed symptoms such as skin ulcers, infections, and suppurations, which seriously affect their quality of life. These patients are more likely to seek surgical treatment to improve their quality of life. For them, local surgical treatment required the resection of the tumor in addition to removing the involved skin, soft tissue, and chest muscle. This leaves a large chest wall defect. However, in some breast centers with limited resources, most surgeons did not have microsurgical skills, so it is difficult for them to find a way to cover chest defect after radical breast surgery.

Previously, different types of flaps had been used to repair the defect in the chest wall. However, the clinical effect was unsatisfactory. Bonomir reported [5] the use of a VY thoracoabdominal perforator flap for LABC chest wall reconstruction. Shoham proposed that [6] a reconstruction method of the chest wall using a latissimus dorsi combined with rectus abdominis muscle flap. Pelzer [7] designed a combined ALT/TFL flap to cover the soft tissue of the large thoracic defect. Gassman reported that [8] the application of a superficial and deep inferior superior abdominal artery in combination with a perforator flap for breast reconstruction. But, due to the complex procedure, longer recovery time, skin grafting, and large trauma, these operations affected the follow-up treatment time. Moreover, in some breast centers with limited resources, it was difficult for surgeons to master microsurgical techniques in a short time. In order to achieve better results, it is a challenge for surgeons to select a safe, simple procedure with less injury and faster recovery.

Tansini in 1896 proposed that the latissimus dorsi flap operation could be used to repair surgical defects after satisfactory tumor resection. Its clinical application in repairing chest wall defects had been commonly used. The advantages of the latissimus dorsi for chest wall defects [9-11] were as 
follows: (1) Latissimus dorsi was located in the upper part of the body, which was close to the receiver region. It could be positioned to the area along the subcutaneous tunnel, and did not pull the vascular pedicle; (2) the supply of the latissimus dorsi blood vessels line was unchanged, which was convenient for the surgeon; (3) the latissimus dorsi and its surrounding tissues were large and could be used to repair large defects; (4) latissimus dorsi myocutaneous flap could be removed without affecting physical function; (5) the latissimus dorsi had a rich blood supply; hence, necrosis was not a concern; (6) the procedure was simple and easy to perform. Based on the design of kiss flap, the traditional latissimus dorsi flap was improved. The latissimus dorsi kiss flap overcome the limitation of the traditional flap width and ensured that the donor skin can be closed to avoid complications that arise from skin grafts. Latissimus dorsi kiss flap met most of the requirements for chest wall reconstruction after mastectomy and had the advantages of a high flap success rate, minimal injury, fast recovery time, and an easy procedure. The kiss flap was ideal for use in resource-limited breast centers because surgeons did not need to master microsurgical techniques, so they could master the kiss flap in a short time.

In this report, we summarized some of our experience regarding the application of the latissimus dorsi kiss flap for the reconstruction of chest wall defects. The kiss flap avoided some surgical complications, such as flap necrosis and skin grafting. Accurate preoperative design was essential for a successful surgery. The surgeon needed to accurately delineate the latissimus dorsi. Both flaps should be mostly located on the latissimus dorsi, and otherwise, partial flap necrosis might occur. The angle between the two flaps should not be too large or too small, preferably 60-90 degrees, to reduce complications in the donor area. If the diameter of the donor defect on the back was greater than $8 \mathrm{~cm}$, a flap would be designed for suture to avoid skin grafts. In addition, postoperative care was also critical for flap survival and donor skin healing. Some of the important points from our experience performing the procedure included: (1) Within 3 days after surgery, it was recommended to observe color of the flap every $2 \mathrm{~h}$; if congestion occurred, alcohol gauze would be placed on the flap, which could dilated the blood vessels supplying the flap; (2) the patient should be kept in the lateral position for 1 week to avoid compression of kiss flap and donor area; (3) within 1-week postsurgery, anticoagulants and vasodilating drugs can be administered to maintain the blood supply of the flap; (4) in addition, low molecular weight heparin (LMWH) could be used to prevent thrombosis of the pedicle of the flap.

In summary, the application of latissimus dorsi kiss flap for the reconstruction of the chest wall after a mastectomy was a safe and reliable procedure. The advantages included minimal injury, fast recovery time, and easy to perform. The latissimus dorsi kiss flap had been satisfactorily performed to repair defects in the chest wall after breast tumor resection previously [12-14]. Compared to traditional latissimus dorsi flap, the latissimus dorsi kiss flap had a satisfactory clinical effect and could effectively reduce the need for re-surgery. This effectively improved the quality of life in patients. In order to the success of procedure, it was critical to perform a systematic and comprehensive assessment of the patient to minimize or eliminate any contraindication. The advantages of applying pedicled latissimus dorsi kiss flap to repair chest wall defect after breast tumor resection include high success rate, small injury, and fast recovery time. This type of operation is worthy of clinical promotion.

\section{Declarations}

Ethics Approval The authors are accountable for all aspects of the work in ensuring that questions related to the accuracy or integrity of any part of the work are appropriately investigated and resolved. Written informed consent was obtained from the patient for publication of this manuscript and any accompanying images. The study protocol was approved by the Medical Ethics Committee of Affiliated Hospital of Guangdong Medical University.

Conflict of Interest The authors declare no competing interests.

Open Access This article is licensed under a Creative Commons Attribution 4.0 International License, which permits use, sharing, adaptation, distribution and reproduction in any medium or format, as long as you give appropriate credit to the original author(s) and the source, provide a link to the Creative Commons licence, and indicate if changes were made. The images or other third party material in this article are included in the article's Creative Commons licence, unless indicated otherwise in a credit line to the material. If material is not included in the article's Creative Commons licence and your intended use is not permitted by statutory regulation or exceeds the permitted use, you will need to obtain permission directly from the copyright holder. To view a copy of this licence, visit http://creativecommons.org/licenses/by/4.0/.

\section{References}

1. Wormann B (2017) Breast cancer: basics, screening, diagnostics and treatment. Med Monatsschr Pharm 40(2):55-64

2. Jia Song, Yong et al (2019) Using KISS flaps in a chest wall reconstruction after mastectomy for locally advanced breast cancer: A new technique:[J]. Surg Innov 27(1):5-10

3. Liu JS (2010) Clinical application of laps dorsi musculocutaneous flap in repairing soft tissue defects of limbs. Jilin Medicine 31(09):1160-1161

4. Zhang Y (2009) Breast reconstruction and anterior axillary fold reconstruction of the island latissimus dorsi musculocutaneous flap after radical mastectomy. Chin J Reconstr Surg 23(03):379-380

5. Bonomi, Stefano, Salval et al (2012) Autologous latissimus dorsi flap as an alternative to free abdomen-based flap for breast reconstruction in the morbidly obese.[J]. Plast Reconstr Surg 129(2):357e-358e 
6. Shoham Y (2013) Immediate reconstruction of the chest wall by latissimus dorsi and vertical rectus abdominis musculocutaneous flaps after radical mastectomy for a huge pleomorphic liposarcoma. J Plast Surg Hand Surg 47(2):152-154

7. Pelzer M (2014) Combined split ALT/TFL flap for soft tissue coverage in large thoracical defects. Chirurg 85(1):42-45

8. Gassman AA (2018) The superficial and deep inferior epigastric artery composite perforator flap for breast reconstruction: a case report. Microsurgery 38(7):799-803

9. Chu-Yu HE, Xing-An LU, Tang JY et al (2004) Transplantation of free latissimus dorsi myocutaneous flaps for craniomaxillofacial reconstruction[J]. Chin J Plast Surg Burns

10. Elliott LF, Raffel B, Wade J (1989) Segmental Latissimus Dorsi Free Flap: Clinical Applications. Ann Plast Surg 3:231-238

11. Schwabegger AH, Harpf C, Rainer C (2003) Muscle-sparing latissimus dorsi myocutaneous flap with maintenance of muscle innervation, function, and aesthetic appearance of the donor site. Plast Reconstr Surg 111(4):1407-11
12. De Lorenzi F, Corso G, Botta F (2020) Immediate breast reconstruction with latissimus dorsi flap for patients with local recurrence of breast cancer. Eur J Surg Oncol 46(6):1013-1020

13. Yamada A, Narui K, Satake T (2020) Long-term outcomes of immediate autologous breast reconstruction for breast cancer patients. J Surg Res 251:78-84

14. Santosa KB, Qi J, Kim HM (2016) Effect of patient age on outcomes in breast reconstruction: results from a multicenter prospective study. J Am Coll Surg 223(6):745-754

Publisher's Note Springer Nature remains neutral with regard to jurisdictional claims in published maps and institutional affiliations. 